\title{
Tempo de recuperação entre a pesagem e o início das lutas em competições de judô do Estado de São Paulo
}

CDD. 20.ed. 796.027

796.81

\author{
Guilherme Giannini ARTIOLI* \\ Emerson FRANCHINI* \\ Marina Yazigi SOLIS* \\ Marina FUCHS* \\ Mariane TAKESIAN* \\ Sandro Henrique MENDES* \\ Bruno GUALANO* \\ Antonio Herbert LANCHA J UNIOR* \\ *Escola de Educa- \\ ção Física e Esporte, \\ Universidade de São \\ Paulo.
}

\section{Resumo}

A perda rápida de peso é altamente prevalente entre atletas de luta. No judô, há um periodo entre a pesagem e 0 início da competição no qual atletas podem se recuperar da perda de peso. Apesar desse tempo ser determinante para o desempenho, nenhum estudo avaliou seu padrão de duração. Este estudo objetivou determinar o padrão de duração do tempo entre a pesagem e o início das lutas em competições oficiais de judô. Foram analisados 117 atletas do sexo masculino (classes juvenil, júnior e sênior) durante duas competições oficiais. Registraram-se o horário de término da pesagem e do início da primeira luta de cada atleta. 0 tempo médio de recuperação foi de aproximadamente quatro horas. A maior parte dos atletas teve aproximadamente 2,5 - 5 horas entre a pesagem e 0 início das lutas. 0 periodo para a classe sênior foi significantemente maior do que o das classes júnior e juvenil $(p<0,01)$ e $o$ da classe júnior foi significantemente maior do que o da classe juvenil $(p<0,01)$. Conclui-se que os tempos de recuperação aqui registrados são provavelmente padrões para competições de mesmo porte e esquema organizacional, embora os tempos específicos para as classes etárias possam se modificar. 0 período que a maioria dos atletas teve para recuperar-se é suficiente para adequada ingestão de alimentos e líquidos, o que minimiza o impacto da perda de peso sobre o desempenho e estimula a pratica de métodos agressivos de perda rápida de peso.

UnIteRMos: Perda de peso; Tempo de recuperação; Pesagem; Artes marciais.

\section{Introdução}

O judô é uma modalidade olímpica na qual os atletas competem em categorias divididas de acordo com o peso corporal. O objetivo de tal divisão é equilibrar as disputas em termos de peso corporal, força e velocidade. Apesar disso, foi demonstrado que a maioria dos competidores de judô reduz significativa quantidade de peso poucos dias antes das competiçóes, com o intuito de enquadrar-se em categorias mais leves do que a correspondente a seu peso habitual (ARTIOLI, Gualano, Franchini, Scagliusi, Takesian, Fuchs \& LANCHA JUNIOR, 2010). Ao adotar tal estratégia, os atletas acreditam obter vantagem competitiva uma vez que, teoricamente, irão enfrentar adversários mais leves e fracos (ArTioli, Franchini \& LANCHA JUNIOR, 2006). O mesmo tipo de comportamento tem sido extensivamente descrito em atletas de outras modalidades cujas competições são também divididas em categorias de peso, como é o caso da luta olímpica (Kiningham \& Gorenflo, 2001; Oppliger, Utter, SCott, Dick \& Klossner, 2006; STEEN \& Brownell, 1990). Para reduzir o peso em um curto período de tempo, os atletas utilizam uma série de estratégias agressivas, tais como: restrição 
da ingestão de líquidos e alimentos, uso de roupas de plástico ou borracha para indução de sudorese, prática de exercícios em locais quentes, aumento da quantidade de exercícios praticados, uso de laxantes e diuréticos ou até mesmo indução de vômitos (Artioli et al., 2010; STEEN \& Brownell, 1990).

A literatura científica tem sido unânime em demonstrar os efeitos prejudiciais dessas práticas sobre diversos parâmetros relacionados à saúde. Existem relatos de que a perda rápida de peso agudamente reduz a densidade óssea (ProutEau, Pelle, Collomp, Benhamou \& Courteix, 2006), afeta funções cognitivas (CHOma, Sforzo \& Keller, 1998), aumenta as chances de desenvolvimento de transtornos alimentares (OpPliger, LANDrY, Foster \& LAMBreChT, 1998), deprime o sistema imune (Imai, Seki, Dobashi, Ohkawa, Habu \& Hiraide, 2002; Ohta, NakajI, Suzuki, Totsuka, UMEDA \& SugaWARA, 2002), promove desequilíbrios hormonais (MCMurRAY, Proctor \& WiLsON, 1991; Roemmich \& Sinning, 1997), pode causar hipertermia e até mesmo morte (SALTin, 1964). De fato, em 1997 três mortes de jovens lutadores norteamericanos foram atribuídas à hipertermia causada pela perda rápida de peso (Centers For Disease Control And Prevention, 1998).

Embora o impacto sobre diversos sistemas fisiológicos esteja bem descrito, os possíveis efeitos da perda rápida de peso sobre o desempenho em lutas ainda permanece incerto e controverso. Alguns estudos demonstraram que a redução rápida de peso prejudica o desempenho em exercícios de alta intensidade e curta duração (Filaire, Maso, Degoutte, Jouanel \& LAC, 2001; HickNer, HorsWILl, WelKer, SCOTt,

\section{Metodologia}

Foram observadas duas competições oficiais de judô ocorridas na cidade de São Paulo durante o ano de 2007 (campeonatos metropolitano e paulistano). Tratam-se, portanto, de competições oficiais de nível regional. Mais especificamente, o campeonato metropolitano é aberto para qualquer atleta federado pela cidade de São Paulo e o paulistano é fechado para os melhores colocados do campeonato metropolitano. Neste estudo observacional, os pesquisadores, nos dias das competições analisadas, ficaram posicionados na saída da sala de pesagem durante todo o período oficial da mesma. Ao sair da sala, atletas selecionados ao acaso eram abordados
Roemmich \& Costill, 1991). Outros, por sua vez, falharam em demonstrar tal efeito (ARTIOLI et al., 2010; Fogelholm, Koskinen, LaAKso, Rankinen \& Ruokonen, 1993; Horswill, Hickner, Scott, Costill \& Gould, 1990). Todavia, as investigações que verificaram queda no desempenho não permitiram que os atletas se recuperassem após a simulação da pesagem (Filaire, et al., 2001; HicKNER et al., 1991). Complementarmente, as investigações que permitiram intervalo inferior a três horas após a pesagem também observaram queda no rendimento, ao passo que as que permitiram intervalos próximos a cinco horas ou mais não observaram efeito da perda rápida de peso sobre o desempenho anaeróbio (para revisão, ver ARTioli, Franchini \& LANCHA JUNIOR, 2006).

Com base nesses achados, pode-se afirmar que o tempo de recuperação após a pesagem tem grande relevância para o desempenho de atletas que reduzem peso e deve ser levado em consideração quando da elaboração da melhor estratégia de controle do peso. Embora seja bem conhecido que todas as competições oficiais nacionais e internacionais apresentem um lapso de tempo entre a pesagem e o início das lutas, nenhum estudo propôs-se a caracterizar o padrão de duração deste intervalo. Considerando-se a relevância desse dado, tanto para a criação de protocolos de pesquisa que investiguem a perda de peso simulando situaçóes competitivas reais quanto para definição da melhor estratégia nutricional durante esse período, o presente estudo teve como objetivo identificar o padrão de duração dos intervalos entre a pesagem e o início das lutas em competições oficiais.

e, após a confirmação de que sua pesagem acabara de ser oficialmente realizada, tinham seus nomes anotados assim como a hora exata de sua saída. Neste estudo, assumimos que a hora de saída da sala poderia ser considerada como a hora em que a pesagem fora efetuada.

Ao início das lutas, os pesquisadores se posicionaram próximos à fila pela qual os atletas entravam na área de combate, registrando a hora exata da primeira luta de cada um dos atletas que haviam sido previamente abordados. Deste modo, foi possível, ao computar a hora da pesagem e a hora do início das lutas, calcular o intervalo de recuperação após a pesagem. 


\section{Amostra}

Foram incluídos na análise 117 atletas do sexo masculino das categorias juvenil (15 a 16 anos de idade; $\mathrm{n}=35$ ), júnior ( 17 a 19 anos de idade; $\mathrm{n}=43$ ) e sênior (20 ou mais anos de idade; $n=49$ ). Uma vez que o total de atletas, considerando-se as três categorias incluídas neste estudo, que participaram nos dois eventos foi de 436, nossa amostra foi constituída por $27 \%$ do total de participantes. Todos os atletas foram informados sobre os objetivos do estudo e sobre os procedimentos necessários antes de assinarem o termo de consentimento livre e esclarecido. No caso de menores de 18 anos, o consentimento foi obtido dos pais ou responsáveis legais. Os procedimentos adotados neste estudo foram aprovados pelo Comitê de Ética em Pesquisa da Escola de Educação Física e Esporte da Universidade de São Paulo.

\section{Análises estatísticas}

Foram realizadas análises descritivas (média \pm desvio padrão e intervalo) e de frequência. Para comparação dos dados obtidos entre os três diferentes grupos de atletas (juvenil, júnior e sênior), foi conduzida análise General Linear Model seguida pelo teste "post-hoc" de Tukey, quando necessário. Todos os requisitos do modelo foram testados e satisfeitos (isto é, distribuição normal dos dados, verificada pelos valores de "skewness" e "kurtosis" com valores entre $-1,0$ e 1,0 , e homogeneidade, verificada pelo teste de Levene). Todas as análises foram realizadas com o auxílio do programa SPSS 15. O nível de significância previamente adotado foi de $5 \%$.

\section{Resultados}

O período médio de recuperação foi de, aproximadamente, quatro horas (TABELA 1). A comparação entre os grupos demonstrou efeito principal $(\mathrm{F}=235,9 ; \mathrm{p}<0,0001$; eta quadrado = 0,801). A análise "post-hoc" revelou que os atletas da classe sênior tiveram um período de recuperação mais longo do que aqueles da classe júnior $(\mathrm{p}<0,0001)$. Os atletas juniores, por sua vez, puderam recuperar-se por um período mais longo do que os juvenis $(\mathrm{p}<0,0001)$ (TABELA 1). A análise de frequência revelou que o tempo de recuperação para a maioria dos atletas variou de 150 a 300 minutos ( $-2,5$ a 5 horas) (FIGURA 1 ).

TABELA 1 - Tempo de recuperação (min) entre pesagem e início das lutas.

\begin{tabular}{lccc}
\hline & Média & Desvio Padrão & Variação \\
\hline Juvenil & 163 & 12 & $124-182$ \\
Júnior & $227^{*}$ & 29 & $168-283$ \\
Sênior & $294^{*}$ & 31 & $253-378$ \\
Geral & 230 & 58 & $124-378$ \\
\hline
\end{tabular}

*significantemente diferente de juvenil +significantemente diferente de júnior.

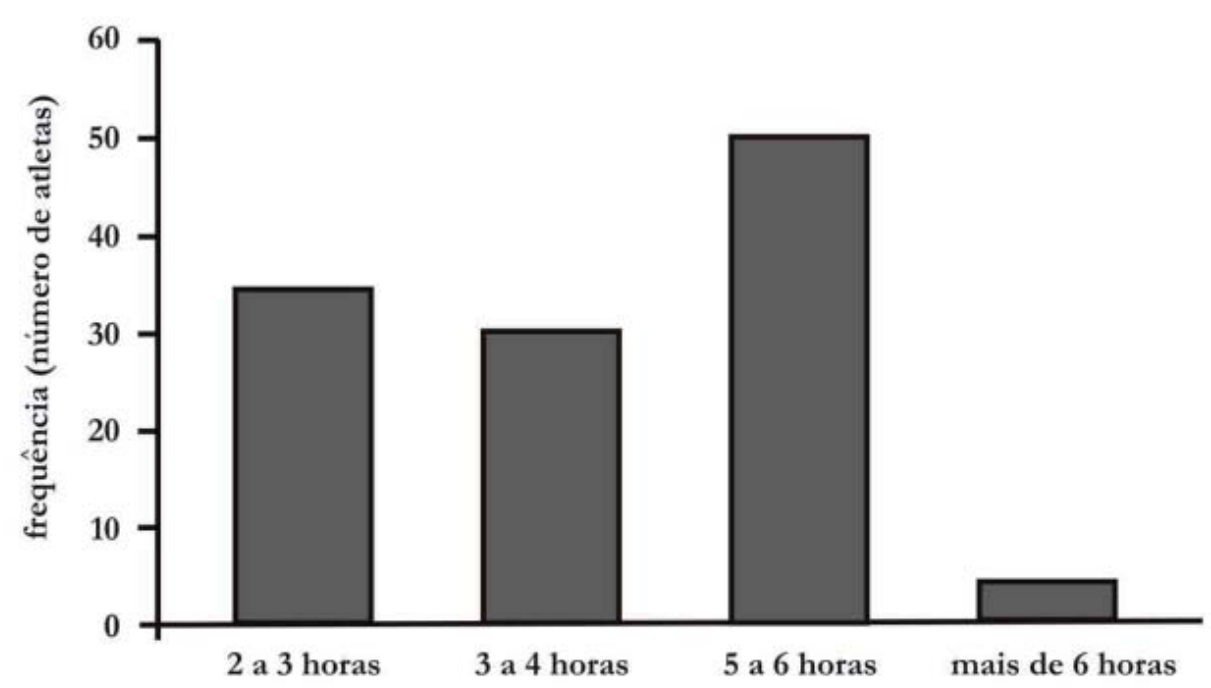

FIGURA 1 - Análise de frequência do tempo de recuperação entre pesagem e início das lutas (n = 117). 


\section{Discussão}

É bem conhecido que as competiçóes oficiais de judô, tanto em nível nacional quanto internacional, apresentam um período de tempo, após a pesagem oficial, no qual os atletas que estejam reduzindo peso podem se realimentar e reidratar, recuperando-se dos efeitos da diminuição do peso. Diversas evidências indicam que a recuperação do desempenho após a perda de peso depende fortemente da duração do período de recuperação (ARTiOli et al., 2010; FogelHolm et al, 1993; Horswill et al., 1990; Rankin, Ocel \& Craft, 1996; Vittasalo, Kyrolainen, Bosco \& Alen, 1987), muito embora fatores como composição da dieta também exerçam papel relevante (FINN, DOLGENER \& Williams, 2004; HorsWILl et al., 1990; MCMurRAY, Proctor \& Wilson, 1991). Logo, a caracterização do padrão de tempo entre a pesagem e o início das lutas é fundamental para o planejamento da estratégia mais adequada de controle de peso. Adicionalmente, o conhecimento dessa variável pode certamente contribuir para que futuros estudos sobre perda de peso possam utilizar protocolos mais próximos de situações competitivas reais. Ao nosso conhecimento, nenhum estudo até então havia caracterizado o tempo de recuperação entre a pesagem e a luta.

Os dados do presente estudo demonstram que o tempo médio de recuperação é de aproximadamente quatro horas e que os períodos mais frequentes variam entre três e cinco horas. Isso significa que a maior parte dos atletas tem tempo suficiente para realimentar-se e reidratar-se de modo a não sentir efeitos negativos da perda rápida de peso sobre o desempenho (ARTIOLI, Iglesias, Franchini, Gualano, Kashiwagura, Solis, Benatti, Fuchs, Lancha Junior, 2010). Esse fato está de acordo com a percepção que os próprios atletas têm de que reduzir peso para lutar em uma categoria mais leve pode proporcionar vantagem competitiva (Artioli et al., 2010; STEEN \& Brownell, 1990). Isso provavelmente estimula um grande número de atletas a optar por fazer uso de estratégias agressivas de perda rápida de peso, o que pode ter efeitos adversos bastante importantes sobre a saúde ou até mesmo resultar em morte (CDC, 1998). Consequentemente, a redução do intervalo entre pesagem e início da competição é uma importante medida que deve ser adotada pelos órgãos que organizam o esporte para diminuir a prevalência da perda rápida de peso entre competidores. De fato, a redução desse intervalo foi uma das medidas-chave tomada pela NCAA (do inglês, National Collegiate Athletic Association, entidade que regula a luta olímpica colegial e universitária nos Estados Unidos) em seu conjunto de novas regras implantadas em 1998-1999 que visavam diminuir a prevalência e intensidade da perda rápida de peso e que se mostraram bastante efetivas (Oppliger et al., 2006).

Um achado interessante deste estudo foi que os tempos médios de intervalo foram significantemente diferente entre as três diferentes classes etárias analisadas. Obviamente, esse fato ocorreu porque, no caso específico das competições aqui analisadas, a classe juvenil iniciou a competição antes da classe júnior, que por sua vez, iniciou antes da sênior. Logo, isso não deve ser interpretado de maneira restrita e o mesmo padrão observado provavelmente não se repetirá em qualquer competição, já que é comum que as entidades organizadoras alterem a ordem das classes etárias que iniciarão a competição. Entretanto, pode-se especular que os tempos médios de recuperação sejam, aproximadamente, os mesmos observados neste estudo para qualquer classe que inicie a competição nas respectivas ordens cronológicas. A importância disso está no fato de ser possível prever o tempo aproximado de recuperação que um dado atleta terá desde que se saiba em qual ordem a classe etária a que pertence iniciará a competição, o que permitiria um melhor planejamento de como utilizar esse tempo para maximizar o desempenho na competição. Possivelmente, tal previsão será mais consistente para as classes que lutarem no início da competição. Deve-se ressaltar que tal semelhança só ocorrerá nas competições cujo porte e esquema organizacional sejam similares às analisadas no presente estudo. Todavia, em competiçōes oficiais promovidas no Estado de São Paulo, essas variáveis provavelmente não sofrerão grandes modificações, $\mathrm{o}$ que facilitaria a previsão do intervalo de recuperação baseada nos dados aqui relatados.

Em suma, este trabalho demonstrou que o período médio de recuperação entre a pesagem e o início das lutas em competições de judô é de, aproximadamente, quatro horas. Períodos variando entre, aproximadamente, 2,5 e cinco horas são os mais frequentemente observados, sendo que a primeira classe a competir terá cerca de 2,5 horas, a segunda terá cerca de quatro horas e a terceira terá cerca de cinco horas de recuperação. Os dados deste estudo provavelmente se repetirão em competiçôes cujo porte e esquema de organização sejam similares aos das competições observadas. Por fim, é imprescindível destacar que os tempos de recuperação observados no 
presente estudo incentivam a rápida perda de peso, cuja prática é sabidamente associada a diversos efeitos prejudiciais à saúde. Assim, recomenda-se fortemente que as normas que regulamentam os períodos de recuperação sejam imediatamente revisadas pelas entidades competentes.

\begin{abstract}
Recovery time between weigh-in and first match in State level judo competitions

Rapid weight loss is highly prevalent among combat sport athletes. After the weigh-in, there is a period in which athletes can refeed and rehydrate before the combats. The length of this recovery period is determinant for performance in the subsequent combats. No study, however, has determined the time patterns of such period. The purpose of this study was to determine the patterns of recovery time between the weigh-in and the first combats during judo competitions. One hundred and seventeen juvenile, junior and senior male athletes were analyzed during two São Paulo state competitions. The time at which each athlete has finished the weight-in and the time at which they have started the first combat were recorded and then the recovery period between weigh-in and combats was calculated. Average recovery time was approximately four hours. Most athletes had a 2.5 to 5-hour recovery time between the weigh-in and the first combat. Senior athletes had a significant longer recovery time compared to junior and juvenile $(p<0.001)$. Junior athletes also had a significant longer recovery time in comparison to juvenile athletes $(p<0.001)$. In conclusion, the patterns for recovery time presented in this study are likely to be a standard if competitions of similar size and organization are considered. Recovery period for the majority of athletes is enough to allow them to refeed and rehydrate, so the impact of weight loss on performance would be minimal. This can stimulate athletes to engage in potentially harmful rapid weight loss procedures.
\end{abstract}

UnItERMS: Weight loss; Recovery time; Weigh-in; Martial arts.

\title{
Notas
}

Guilherme Giannini Artioli é bolsista CNPq e Marina Yazigi Solis é bolsista CAPES.

Este estudo foi financiado pela FAPESP (\# 06/51293-4).

\section{Referências}

ARTIOLI, G.G.; FRANCHINI, E.; LANCHA JUNIOR, A.H. Perda rápida de peso em esportes de combate de domínio: revisão e recomendações aplicadas. Revista Brasileira de Cineantropometria e Desempenho Humano, Florianópolis, v.8, p.92-101, 2006. ARTIOLI, G.G.; GUALANO, B.; FRANCHINI, E.; SCAGLIUSI, F.B.; TAKESIAN, M.; FUCHS, M. LANCHA JR, A.H. Prevalence, magnitude and methods of rapid weight loss among judo competitors. Medicine and Science in Sports and Exercise, Madison, v.42, n.3, p.436-42, 2010.

ARTIOLI, G.G.; IGLESIAS, R.T.; FRANCHINI, E.; KASHIWAGURA, D.B.; SOLIS, M.Y.; BENATTI, F.B.; FUCHS, M.; LANCHA JUNIOR, A.H. Rapid weight loss followed by recovery time does not affect judo-related performance. Journal of Sports Sciences, London, v.28, n.1, p.21-32, 2010.

CENTERS FOR DISEASE CONTROL AND PREVENTION. Hyperthermia and dehydration-related deaths associated with intentional rapid weight loss in three collegiate wrestlers - North Carolina, Wisconsin, and Michigan, NovemberDecember 1997. The Journal of the American Medical Association, Chicago, v.18, n.11, p.824-25, 1998.

CHOMA, C.W.; SFORZO, G.A.; KELLER, B.A. Impact of rapid weight loss on cognitive function in collegiate wrestlers. Medicine and Science in Sports and Exercise, Madison, v.30, p.746-749, 1998.

FILAIRE, E.; MASO, F.; DEGOUTTE, F.; JOUANEL, P.; LAC, G. Food restriction, performance, psychological state and lipid values in judo athletes. International Journal of Sports Medicine, Stuttgart, v.22, p.454-459, 2001. 
FINN, K.J.; DOLGENER, F.A.; WILLIAMS, R.B. Effects of carbohydrate refeeding on physiological responses and psychological and physical performance following acute weight reduction in collegiate wrestlers. Journal of Strength and Conditioning Research, Lincoln, v.18, p.328-333, 2004.

FOGELHOLM, G.M.; KOSKINEN, R.; LAAKSO, J.; RANKINEN, T.; RUOKONEN, I. Gradual and rapid weight loss: effects on nutrition and performance in male athletes. Medicine and Science in Sports and Exercise, Madison, v.25, p.371-7, 1993. HICKNER, R.C.; HORSWILL, C.A.; WELKER, J.M.; SCOTT, J.; ROEMMICH, J.N.; COSTILL, D.L. Test development for the study of physical performance in wrestlers following weight loss. International Journal of Sports Medicine, Stuttgart, v.12, p.557-62, 1991.

HORSWILL, C.A.; HICKNER, R.C.; SCOTT, J.R.; COSTILL, D.L.; GOULD, D. Weight loss, dietary carbohydrate modifications, and high intensity, physical performance. Medicine and Science in Sports and Exercise, Madison, v.22, p.470-6, 1990. IMAI, T.; SEKI, S.; DOBASHI, H.; OHKAWA, T.; HABU, Y.; HIRAIDE, H. Effect of weight loss on T-cell receptormediated T-cell function in elite athletes. Medicine and Science in Sports and Exercise, Madison, v.34, p.245-50, 2002. KININGHAM, R.B.; GORENFLO, D.W. Weight loss methods of high school wrestlers. Medicine and Science in Sports and Exercise, Madison, v.33, n.5, p.810-3, 2001.

McMURRAY, R.G.; PROCTOR, C.R.; WILSON, W.L. Effect of caloric deficit and dietary manipulation on aerobic and anaerobic exercise. International Journal of Sports Medicine, Stuttgart, v.12, p.167-72, 1991.

OHTA, S.; NAKAJI, S.; SUZUKI, K.; TOTSUKA, M.; UMEDA, T.; SUGAWARA, K. Depressed humoral immunity after weight reduction in competitive judoists. Luminescence, Malden, v.17, p.150-7, 2002.

OPPLIGER, R.A.; LANDRY, G.L.; FOSTER, S.W.; LAMBRECHT, A.C. Wisconsin minimum weight program reduces weight-cutting practices of high school wrestlers. The Clinical Journal Sports Medicine, Calgary, v.8, p.26-31, 1998.

OPPLIGER, R.A.; UTTER, A.C.; SCOTT, J.R.; DICK, R.W.; KLOSSNER, D. NCAA rule change improves weight loss among national championship wrestlers. Medicine and Science in Sports and Exercise, Madison, v.38, p.963-70, 2006. PROUTEAU, S.; PELLE, A.; COLLOMP, K.; BENHAMOU, L.; COURTEIX, D. Bone density in elite judoists and effects of weight cycling on bone metabolic balance. Medicine and Science in Sports and Exercise, Madison, v.38, p.694-700, 2006. RANKIN, J.W.; OCEL, J.V.; CRAFT, L.L. Effect of weight loss and refeeding diet composition on anaerobic performance in wrestlers. Medicine and Science in Sports and Exercise, Madison, v.28, p.1292-9, 1996.

ROEMMICH, J.N.; SINNING, W.E. Weight loss and wrestling training: effects on growth-related hormones. Journal of Applied Physiology, Bethesda, v.82, p.1760-4, 1997.

SALTIN, B. Circulatory response to submaximal and maximal exercise after thermal dehydration. Journal of Applied Physiology, Bethesda, v.19, p.1125-32, 1964.

STEEN, S.N.; BROWNELL, K.D. Patterns of weight loss and regain in wrestlers: has the tradition changed? Medicine and Science in Sports and Exercise, Madison, v.22, p.762-68, 1990.

VIITASALO, J.T.; KYROLAINEN, H.; BOSCO, C.; ALEN, M. Effects of rapid weight reduction on force production and vertical jumping height. International Journal of Sports Medicine, Stuttgart, v.8, p.281-5, 1987.

\section{Agradecimentos}

Os autores agradecem aos atletas que participaram deste estudo e ao Sr. Antonio Mesquita, da Federação Paulista de Judô, por gentilmente informar o número total de participantes nas competições estudadas.

Agradecemos ao Prof. Dr. Hamilton Roschel pela ajuda na análise estatística.

ENDEREÇO

Guilherme Giannini Artioli

Lab. de Nutrição e Metabolismo Aplicados à Atividade Motora Escola de Educação Física e Esporte - USP

Av. Prof. Mello Moraes, 65

05508-030 - São Paulo - SP - BRASIL

e-mail: artioli@usp.br
Recebido para publicação: 16/03/2011

Revisado: 07/06/2011

Aceito: 13/06/2011 